\title{
Experiment and Optimization of Monitoring Signal Transmission in Underground Coal Mine
}

\author{
Jinhao Zhang ${ }^{1, *}$, Min Chen ${ }^{2}$, Yahui Liu ${ }^{1}$ \\ ${ }^{1}$ China Coal Technology Engineering Group Chongqing Research Institute, Chongqing, 400033, China \\ ${ }^{2}$ Chongqing Keneng Tenior Technician School, Chongqing, 400033, China \\ *Corresponding Author email: 371497416@qq.com
}

Keywords: Monitoring system; signal transmission; optical fiber communication

\begin{abstract}
In order to improve the inspection period of the mine safety monitoring system in coal mine, the underground monitoring substation is divided into two large areas, each of which is equipped with a signal transmission line independently. Two signal transmission lines are converged to the ground safety monitoring center. With the development of science and technology, the mechanization, automation and integration degree of coal mines is constantly improving. In order to increase labor productivity, prevent disasters and accidents, reduce the mortality rate of production activities to the lowest level, and achieve the goal of scientific management. The construction of a reliable, flexible and unimpeded underground coal mine monitoring system has become a necessary construction of modern coal mine construction. A new scheme of current signal acquisition and monitoring for mine power supply system is proposed, and the realization method of software and hardware is emphatically analyzed. This paper combines the mine working environment with optical fiber communication technology, and puts forward the design plan. Based on the reuse technology, the overall design method is given. At the same time, this paper takes into account the special environmental characteristics of coal mine roadway, fiber transmission characteristics, transmission distance, information size and other factors to establish the size of the optical system, working window, signal transmission rate and other overall parameters.
\end{abstract}

\section{Introduction}

Mine safety monitoring system is an important means of modern coal production safety management. It can monitor environmental parameters in real time and provide first-hand information for safety production. China is a big coal resource consuming country, and is also the largest coal producing country in the world. Coal industry is related to our national economy and people's livelihood [1]. At present, there are about 100,000 coal mines in China. The underground power grid monitoring system is composed of ground computer management main station and substation monitoring substation. For a long time, the underground current signal is converted by electromagnetic current transformer, and then transmitted to the ground main station by cable. With the development of the times, the progress of science and technology, the continuous development of hardware technology, in China's coal mine development, to achieve a set of multi-functional automated production [2]. Through the comparative analysis of the measured parameters, technical data are provided for the prevention of disasters and accidents, so as to take preventive measures in advance. At the same time, it can realize automatic alarm, power failure and locking in time, so as to prevent accidents from happening or expanding. The disadvantage of this method is that the transmission process is affected by the downhole harsh environment, especially electromagnetic interference, which will make the signal deviate in the process of measurement and transmission, affecting the reliability of power supply [3]. At the same time, it also affects every link and safety guarantee of the whole mine production system. 


\section{Hardware design of downhole monitoring signal transmission system}

The optical signal transmitted by an optical transmitter attenuates distortion when it passes through the transmission process. The optical receiver can also amplify the distorted signal and restore it as far as possible to the original signal. Optical transmitter is an important part of the light source components, the performance and quality of optical fiber communication system is essentially related to its performance. The key component of optical receiver is semiconductor photodetector. Due to the influence of transmission distance, optical signal attenuation will occur in optical fiber transmission. It is necessary to take some necessary measures to enhance the intensity of the optical signal, so that the escape optical signal can be transmitted further. The realization of optical amplification technology has far-reaching significance in the progress of optical fiber communication. The downhole current signal is collected by coil and converted into digital signal. The photoelectric transmission system converts the digital signal into optical signal and transmits it to the ground main station through optical fiber. The ground master station transforms the optical signal into digital signal and processes it to realize the measurement and monitoring of underground current signal. Although the speed of optical terminals is not the same, the ITU Telecommunication Standardization Organization specifies the code type of the series of Digital interfaces at the base group rate of 2048 kbit/s. As shown in Table 1, the interface code type specified by each bit rate.

Table 1 Interface codes of all bit rates

\begin{tabular}{ccccc}
\hline Group road grade & Primary group & Two group & Three group & Four group \\
\hline Interface rate & 2048 & 8194 & 32776 & 131104 \\
Interface code & HDB & HDB & HDB & CMI \\
\hline
\end{tabular}

The average interference level at each frequency point is calculated by statistical analysis of all the data in the spectrum. The average envelope spectrum of the disturbance voltage on the power line as shown in Fig. 1 can be drawn.

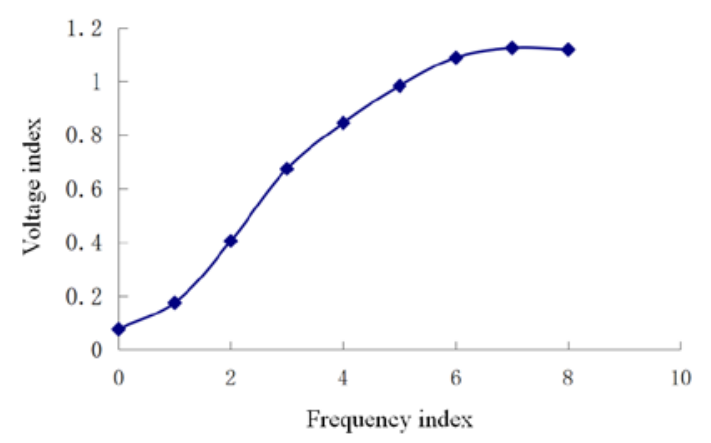

Figure 1 Mean envelope spectrum of disturbance voltage on power line during normal operation of equipment

According to the spectrum of disturbance voltage on the power line of all the measured equipments, the statistical tables shown in Table 2 can be obtained by analyzing the related definitions of characteristic parameters in frequency domain. The statistical data in the table are the basic parameters for studying the frequency domain characteristics of electromagnetic interference, and are the basis for statistical analysis and normality test.

Table 2 Analysis of frequency domain characteristic parameters of power line interference voltage during normal operation of equipment

\begin{tabular}{ccc}
\hline Serial number & Frequency range & Voltage value \\
\hline 1 & $0.05-0.92$ & -45 \\
2 & $0.05-0.56$ & -54 \\
3 & $0.05-0.73$ & -52 \\
4 & $0.05-0.77$ & -49 \\
5 & $0.05-0.85$ & -53 \\
\hline
\end{tabular}


When optical fiber is used as transmission line, the transmitted signal will gradually weaken with the increase of transmission length. In order to make the transmission length longer, it is necessary to restore the weak signal. The application of the chemical converter is an old way to strengthen the optical signal, and there are many disadvantages in this way. Firstly, since different bit rates and signal formats require different regenerators, the regenerator can operate at a given signal bit rate and signal format. Second, the cost of the network is very high, because each channel needs its own matching regenerator. The realization of optical amplification technology has a profound impact on the way forward of optical fiber communication. Optical amplification is the basic method to enhance the optical signal transmission capability. Optical amplifier is an important part of optical fiber communication. It can be used as current amplifier, circuit amplifier and power amplifier. The goal of optical amplifiers is to amplify optical signals. In optical fiber communication, the rapid development of Internet requires that it can share resources, such as PC, which can not be directly connected to the output line of the terminal. Therefore, in order to achieve the purpose of connecting the PC and the optical terminal, the general asynchronous transceiver can be used to realize the parallel-serial conversion between the computer and the serial port.

\section{Experiment and optimization of signal transmission in mine monitoring system}

In complex working environment, although the scope of monitoring is relatively large, more data need to be tested. However, it is necessary to monitor the situation at any time. Therefore, when building the monitoring system, the data transmission and reception speed should be fast enough to meet the conditions that the monitoring personnel can view at any time. Under normal conditions, the concentration of combustible gases such as armour burning in coal mine roadways is below the standard. To test the concentration of these combustible gases, the sensor must also be accurate at low concentrations, i.e. sensitivity separation. It is necessary to continuously develop new sensors with high sensitivity and high performance to complete the testing of various parameters under the mine. Different from the ground requirements, based on the reasons of the mine environment itself, the monitoring system must be easy to install, more convenient to use, and the system can be used for many years after installation, good stability. However, there is much water and humidity in the mine roadway, Dust Haze is serious, humidity is still high, these factors on the monitoring system corrosion damage is more serious. And mine roadway is generally relatively narrow, but also limits the maintenance and repair of equipment. The voltage calculated at the optimal time step is shown in Figure 2. The voltage calculated at the non optimal time step is shown in Figure 3.

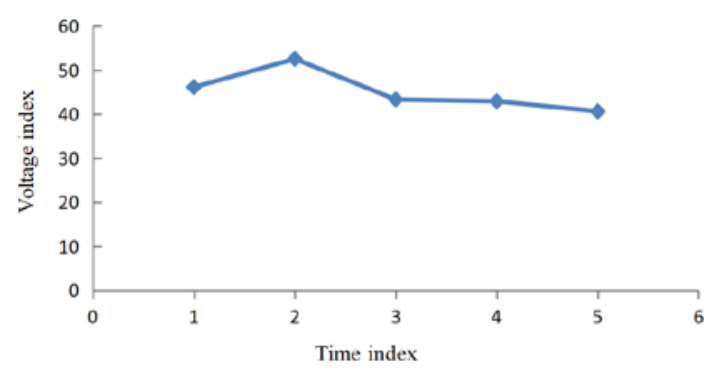

Figure 2 Voltage diagram calculated at optimum time step

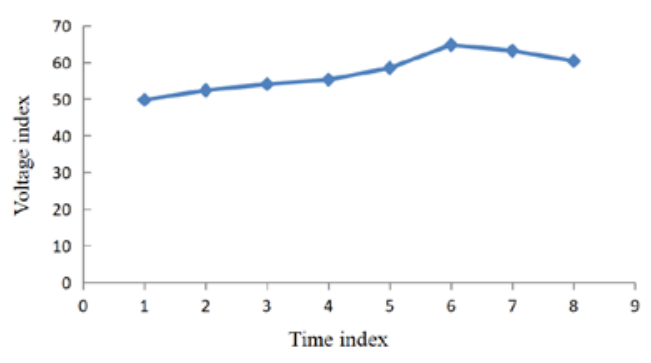

Figure 3 Voltage calculated at non optimal time step 
Mine video surveillance system collects video images of the place under surveillance by video cameras and transmits them to the surveillance room in real time, so that the working conditions of the underground personnel can be known at any time. So that the monitoring staff of the monitoring room can differentiate various on-site situation numbers, make timely judgments and processing, and issue dispatching orders. Realize the visualization of centralized dispatch, realize the safe production of coal mine industry, dispatch and command personnel at any time, and scientific leadership of leaders. Mine video monitoring system consists of: (1) front-end equipment; (2) transmission equipment; (3) control equipment; (4) display equipment; (5) video storage.

The disturbance voltage and the power line in the safety monitoring signal circuit are related to the distance between the safety monitoring signal circuits. According to the peak value of the disturbance voltage corresponding to each different value, Table 3 can be obtained.

Table 3 The interference between the power line and the safety supervision signal is different when the distance is different

\begin{tabular}{cccc}
\hline Distance & 0.1 & 0.5 & 1 \\
\hline A end & 4.69 & 0.61 & 0.12 \\
B end & 5.59 & 0.75 & 0.09 \\
\hline
\end{tabular}

When the mine workers are working in the mine, they must carry the identification card with their own information. When workers enter the mine roadway, they can accurately detect the label of the identification card as long as they are within the monitoring range of the location sub-station. The information given to the workers is then transmitted through the signal transmission system to the ground monitoring for inspection by the monitoring personnel. The two-dimensional rectangular coordinate system is constructed on the location area map, and the relevant parameters of the reference positioning node and the mobile positioning node are loaded. It can survey the actual situation of mine roadway on the spot, and can draw one or more corresponding map of locating area. Each map of locating area is equipped with a coordinate system and draws the coordinates of reference nodes. As shown in Table 4 and Figure 4.

Table 4 Reference node information

\begin{tabular}{ccc}
\hline Reference node & Temperature & Gas concentration (\%) \\
\hline 1 & 32.1 & 0.3 \\
2 & 30.5 & 0.5 \\
\hline
\end{tabular}

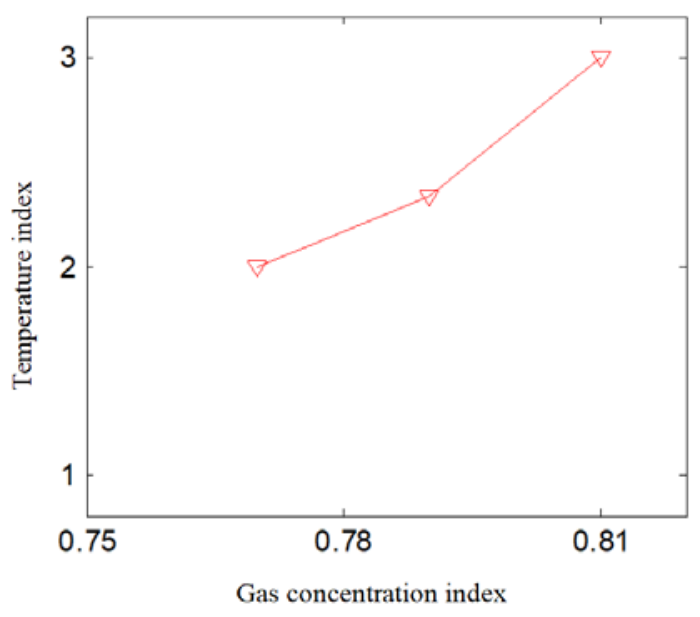

Figure 4 Reference node information

\section{Conclusion}

Now many coal mine production began to use the "digital mine" technology, so the signal transmission system in the coal mine safety system occupies a growing proportion. Considering the 
difference between the working environment in the mine and the ground. In this paper, linear distortion, dispersion, transmission rate, transmission capacity and other factors are considered in the design of signal transmission system. The automatic operation mode of the system has certain significance for saving labor force, saving electric power resources and ensuring coal mine safety production. Therefore, it is very important and necessary to carry out numerical prediction and Research on complex electromagnetic environment in combination with the specific situation of coal mine. For improving the relevant theory of electromagnetic interference, and then effectively guide the safety monitoring system in engineering practice anti-interference design and electromagnetic compatibility immunity evaluation. Ultimately, it is of great theoretical value and practical significance to improve the anti-interference ability of safety supervision system and vigorously guarantee the safety production in coal mines.

\section{References}

[1] Dohare Y S, Maity T, Das P S, et al. Wireless Communication and Environment Monitoring in Underground Coal Mines â $\square$ “ Review[J]. Iete Technical Review, 2015, 32(2):140-150.

[2] Ray D N, Majumder S, Maity A, et al. Design and development of a mobile robot for environment monitoring in underground coal mines [J]. 2015:1-7.

[3] Yang B, Liddell K, Aminossadati S M, et al. Fibre optic based distributed temperature sensing system monitoring of underground coal mine inertisation in Australia[J]. Fiziologicheskiı̌ Zhurnal Sssr Imeni I.m.sechenova, 2015, 55(10):1260-71. 